\title{
La asignatura Informática Industrial y la función del Ingeniero Informático en la Industria
}

\author{
G. Olivares, M. Damas, F. Gómez \\ Departamento de Arquitectura y Tecnología de Computadores. ETSI Informática y de \\ Telecomunicación. Universidad de Granada \\ Granada, España \\ \{gonzalo,mdamas, frgomez\}@ugr.es
}

\begin{abstract}
Resumen. Describimos en este trabajo los objetivos y el desarrollo previsto para la asignatura "Informática Industrial" que se impartirá como optativa en el grado en Ingeniería Informática de la Universidad de Granada, a partir del curso 2013-14. En el artículo se analiza cómo se organiza la docencia teórica y práctica con el fin último de dar a conocer el importante papel del Ingeniero Informático actual en el mundo de la Ingeniería de Control de Procesos Industriales y reflexionar cómo se puede motivar a los alumnos en una disciplina que, sin olvidar los fundamentos de los sistemas de control, debe dirigirse a conseguir que el futuro Ingeniero Informático sepa cuáles van a ser las funciones y competencias que se esperan de un profesional y los conocimientos globales que necesitará para comunicarse con los Ingenieros de Procesos, a la hora de diseñar e implantar Sistemas de Información, Supervisión y Control para la Industria.
\end{abstract}

Palabras Clave: Informática Industrial, Control, Ingeniería Informática, motivación del alumnado.

\begin{abstract}
In this paper we describe the "Industrial Computing" subject that is taught as an elective subject in Computer Engineering degree at the University of Granada. We analyze how the theoretical and practical teaching is organized in order to raise awareness of the important role of the current Computer Engineers in the Industrial Control world and how to motivate students in a discipline that, without forgetting the fundamentals of control systems, must be addressed to ensure that the future engineer knows what will be the functions roles and responsibilities expected of a professional and comprehensive knowledge they need to communicate with engineers industrial process to implement Information, Monitoring and Control Systems for Industry.
\end{abstract}

Keywords: Industrial Computing, Control, Computer Engineering, student motivation. 


\section{Introducción}

La nueva asignatura "Informática Industrial" del grado de Ingeniería Informática de la Universidad de Granada, es una disciplina que se impartirá como optativa a partir del curso 2013-2014 y ha sido planificada basándonos en la experiencia acumulada tras impartir durante varios años las asignaturas "Informática Industrial” de la anterior Ingeniería Técnica Informática y "Sistemas de Control Digital", asignatura de Ingeniería Informática en la Escuela Técnica Superior de Ingenierías Informática y de Telecomunicación de la misma universidad, así como nuestra propia experiencia profesional y empresarial en el ámbito del Control Industrial en las últimas dos décadas. Esta asignatura está íntimamente relacionada, y se complementa adecuadamente, con otra asignatura optativa: "Controladores Lógicos Programables" que se imparte en el mismo curso.

El alumno del Grado en Ingeniería Informática, de partida, piensa que históricamente, e incluso en la actualidad, los únicos ingenieros que trabajan en la automatización y control de procesos son casi exclusivamente Ingenieros Industriales o bien Ingenieros en Electrónica Industrial. Algunas de las funciones del Ingeniero de Control están relacionadas con el diseño, desarrollo e implantación de la instrumentación de control (sensores, actuadores, reguladores,..), con la programación de Controladores Lógicos Programables, con el diseño y configuración de redes locales industriales y buses de campo y también con el desarrollo de las aplicaciones informáticas de monitorización y control que se llevan a cabo en Centros de Control. Todas ellas son funciones que perfectamente puede llevar a cabo el profesional informático con los complementos de formación apropiados.

\section{Motivación}

No es fácil que el alumno deje de pensar que este mundo le es ajeno; casi siempre pensará que su papel profesional potencial se sitúa más bien dentro del equipo de Informáticos de Gestión, trabajando justo en la parte superior de la pirámide CIM (Computer Integrated Manufacturing). Nuestro primer objetivo es hacerle ver que el papel del profesional informático dentro de este sector es esencial, aunque eso sí, tendrá que convivir y entenderse con otros profesionales de otras ramas de la Ingeniería, y tendrá que hablar con propiedad el mismo lenguaje que ellos. Para lograr convencerlo disponemos de un curso de 6 créditos.

Si, según el diccionario de la Real Academia de la Lengua, "la Automática es la disciplina que trata de métodos y procedimientos cuya finalidad es la sustitución del operador humano por un operador artificial en la ejecución de una tarea física y mental previamente programada", este concepto es actualmente mucho más amplio, ya que, si hablamos de la Automática aplicada a la Industria, aparte de hacer énfasis en la sustitución del operador, habría que incluir también otras muchas funciones, tales como: la mejora de la calidad de los productos manufacturados y de las prestaciones de los sistemas a controlar, el desarrollo de métodos de fabricación flexible de acuerdo a las condiciones variantes del mercado, la seguridad global de personas y bienes de producción, el ahorro de costes de materia prima, la supervisión 
en forma jerárquica de todos los procesos y la sincronización de las diferentes áreas de los procesos productivos, desde el nivel de campo hasta el nivel de gestión. El Ingeniero Informático debe jugar aquí un papel esencial, sobre todo en el diseño y programación de los sistemas de control local y distribuido, en la implantación de los sistemas de comunicaciones (buses de campo y redes locales industriales), en la implementación de sistemas de control y supervisión, en el despliegue de servidores y bases de datos, en el diseño de los sistemas SCADA, o en la integración con sistemas ERP.

En la mayoría de las Escuelas de Ingeniería Industrial la asignatura "Informática Industrial" se dirige hacia enfoques de programación, o de Ingeniería de Software, en general. En nuestro caso, aunque la asignatura tenga el mismo nombre, el objetivo es justamente el contrario: se trata de acercar al futuro Graduado en Informática al mundo Industrial, intentando conseguir que pierda el miedo a trabajar en el sector, y ampliando sus horizontes profesionales, haciéndole ver que su vocación se puede abrir a otros ámbitos, más allá de la programación de software de gestión, del desarrollo de aplicaciones web, del mantenimiento de redes y servidores o de la implementación de "apps" para teléfonos y tablets, entre otros.

\subsection{Objetivos de aprendizaje}

En términos generales, como hemos dicho anteriormente, el Ingeniero Informático Industrial tendrá que convivir y formar parte de equipos multidisciplinares (Instrumentistas, Ingenieros de Campo, Ingenieros de Procesos, Ingenieros Eléctricos, o Ingenieros de Producción). Así, los objetivos específicos de aprendizaje son:

- Introducir al alumno en los conceptos básicos sobre sistemas de regulación y control de sistemas lineales.

- Conocer la evolución histórica de la Informática Industrial.

- Aprender a modelar sistemas y procesos, utilizando herramientas software de análisis y simulación.

- Conocer los fundamentos de controladores PID, y aprender a ajustar experimentalmente lazos de regulación.

- Conocer los fundamentos de la arquitectura y de los lenguajes de programación normalizados de Controladores Lógicos Programables.

- Diseñar sistemas de adquisición de datos, supervisión y control.

- Aprender a evaluar los principales sensores, actuadores, sistemas de control, ordenadores industriales y terminales remotas que habitualmente se utilizan en la Industria.

- Conocer los principales buses de campo y redes locales industriales, así como las configuraciones de control distribuido.

- Diseñar y configurar sistemas SCADA para la supervisión y control de plantas industriales.

- Diseñar los elementos de centros de control y arquitecturas de supervisión distribuida

- Tener contacto con aplicaciones reales de automatización industrial. 


\section{Contenidos de la asignatura}

\subsection{Programa de Teoría}

Tras la presentación de la asignatura, se presenta un tema de "Introducción a los Sistemas de Control" destinado a dar al alumno una visión rápida de la materia que se va a estudiar a lo largo del curso, de una manera global y resumida, al objeto de conseguir que el alumno adquiera rápidamente los conocimientos necesarios para iniciar las prácticas experimentales de laboratorio. En este tema se presentan los conceptos de sistemas de regulación en lazo abierto y lazo cerrado, control secuencial y se da una visión del control por computador. A continuación se analizan con algo más de detalle los elementos de un lazo de control: medidores, actuadores, y controladores.

Para finalizar el tema de introducción, se presentan varios ejemplos de sistemas de control reales. Finalizado este tema, el alumno tiene que realizar y presentar un conjunto de ejercicios que le obligarán a hacer una reflexión para identificar los elementos y la arquitectura de diversos sistemas de control mediante el análisis de problemas concretos (regulación de insulina para enfermos diabéticos, control automático de la velocidad de un coche, control del timón de un barco a motor o a vela, identificación de elementos de un vehículo "Segway", estudio de un sistema de control de la climatización del habitáculo de un vehículo, etc ..).

A continuación se introduce al alumno en la teoría de control clásica, estudiando el modelado de sistemas lineales mediante funciones de transferencia y ecuaciones de estado, para posteriormente estudiar la respuesta transitoria de sistemas de primer y segundo orden, y la determinación de estabilidad en sistemas de control continuo. Además se inicia al alumno en el diseño de controladores. En todos los casos, se presentan ejemplos realizados con Matlab y Simulink, para que el alumno entienda mejor los fundamentos teóricos a partir de aplicaciones prácticas. Así, recurrimos al modelado de sistemas de control sencillos pero ilustrativos, tales como a) el control de velocidad de crucero de un coche, b) el análisis del comportamiento térmico de una vivienda, como parte de un sistema domótico, c) la regulación del nivel de un tanque de líquido, y d) el modelado de un péndulo invertido, como fundamento físico del vehículo Segway. La Figura 1, ilustra el modelado de dichos sistemas.

Pasamos a continuación, según el programa diseñado, al estudio de un tema dedicado a "Sensores y Actuadores", desglosado de la siguiente forma:

Fundamentos y clasificación de sensores. Características estáticas y dinámicas. Criterios de selección. Tipos normalizados de señales de salida. Galgas extensiométricas, presión y células de carga. Temperatura (PTC, NTC, Termistores, pirómetros de radiación,..). Posición lineal y angular: codificadores incrementales y absolutos. Nivel y Caudal. Relés, contactores, solenoides. Motores: continua, alterna, de pasos. Actuadores electrohidráulicos y electro-neumáticos. Válvulas. Actuadores térmicos. 

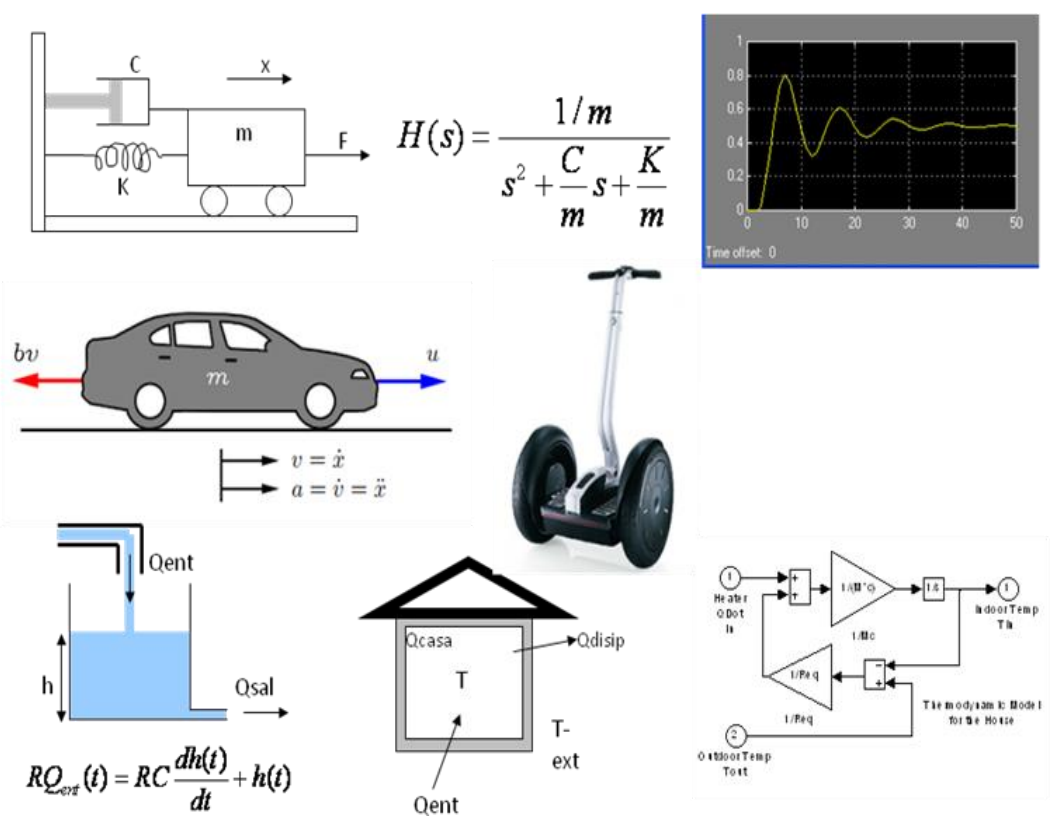

Figura 1. Ejemplos de modelado de sistemas lineales.
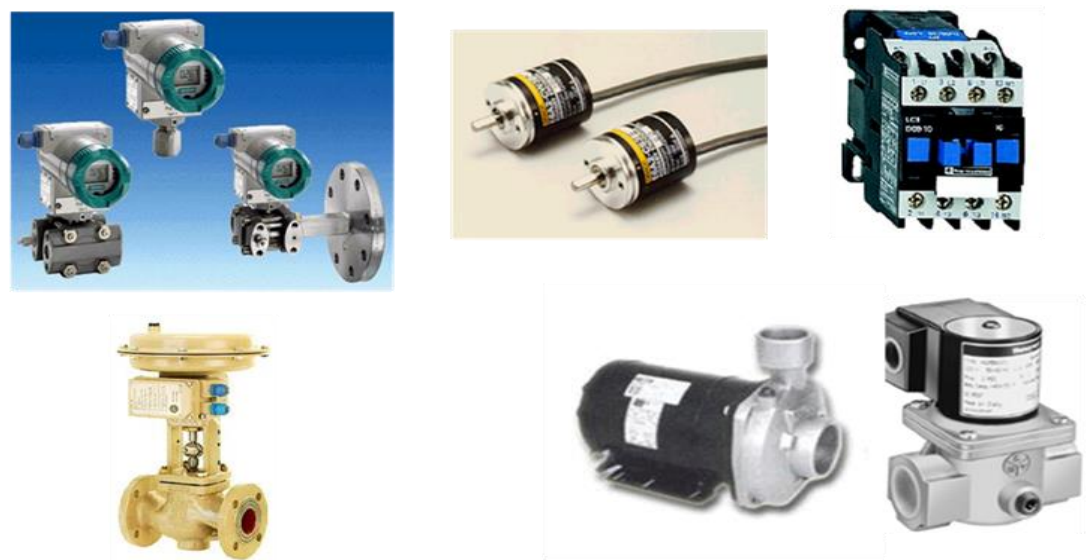

Figura 2. Ejemplos de sensores y actuadores industriales

Además de estudiar los fundamentos de los principales sensores, se pretende conseguir que el alumno pueda identificar los distintos parámetros que le ayudarán a seleccionar adecuadamente la instrumentación que necesitaría para la implementación de sistemas de control (ver figura 2). En todos los casos, una vez revisados los fundamentos de los medidores o actuadores, les presentaremos 
catálogos y documentos de productos de distintos fabricantes para que puedan analizar aspectos tales como: rango de medida, resolución, precisión, ancho de banda, histéresis, condiciones de sobre-rango, requisitos de instalación y mantenimiento, condiciones ambientales de medida, etc.

Más adelante estudiamos con más profundidad las distintas opciones de controladores, según los siguientes apartados:

Fundamentos de control. Controladores "todo-nada" . Controladores continuos. Medida de la calidad de un sistema de control. Métodos de ajuste. Control en cascada. Controladores analógicos. Control secuencial. Control mediante lógica cableada. Controladores Lógicos programables.

Se revisan los principales métodos de ajuste experimental de lazos de regulación y los parámetros que nos permiten diseñar una respuesta transitoria adecuada. Se estudian las características de los reguladores industriales comerciales, y los fundamentos del control secuencial. Tras visualizar algunos vídeos de procesos industriales de control secuencial, se plantean algunos ejemplos de diseño combinacional, para a continuación, transmitirle al alumno la necesidad de utilizar Controladores Lógicos Programables-PLCs. Dado que existe otra asignatura optativa específica en la titulación, se dedica exclusivamente un pequeño apartado del tema a esta materia, dirigido sólo a aquellos alumnos que por cualquier circunstancia no la han elegido. Se estudia de manera global y esquemática la arquitectura y ciclos de funcionamiento de los PLCs, se citan los lenguajes de programación normalizados (IEC 1131-C) y se presentan algunos sencillos ejemplos de programación en SFC, a partir de las especificaciones de diseño.

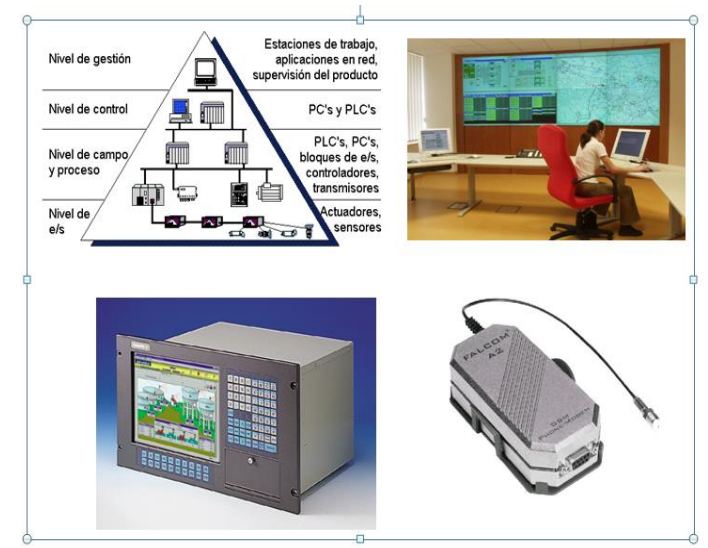

Figura 3. Arquitecturas, sistemas de control, redes y equipos de comunicaciones.

A continuación, una vez asimilados los conocimientos básicos, enfocamos el resto de la asignatura a revisar las principales Arquitecturas, Redes de Control y Sistemas de Comunicaciones Industriales según el siguiente desglose: 
Sistemas en modo supervisión. Control Digital Directo. Control Distribuido. Concepto CIM. Ordenadores Industriales y Periféricos. Centros de Control. Buses de Campo y Redes Locales Industriales.

Actualmente, son muchos los Ingenieros Informáticos que trabajan en el desarrollo de aplicaciones de supervisión, control y adquisición de datos (SCADA"Supervisory Control and Data Acquisition)". Le dedicaremos un apartado específico al estudio de las principales características de este tipo de herramientas de software configurable: creación de esquemas sinópticos, tags, históricos, informes, alarmas, interfaces de comunicaciones vía OPC, etc. Se muestran diversos ejemplos de aplicaciones reales de redes de supervisión de sistemas de abastecimiento de agua, plantas de fabricación de papel, sistemas de bombeo, control integral de una estación de esquí, y control de instalaciones de edificios.
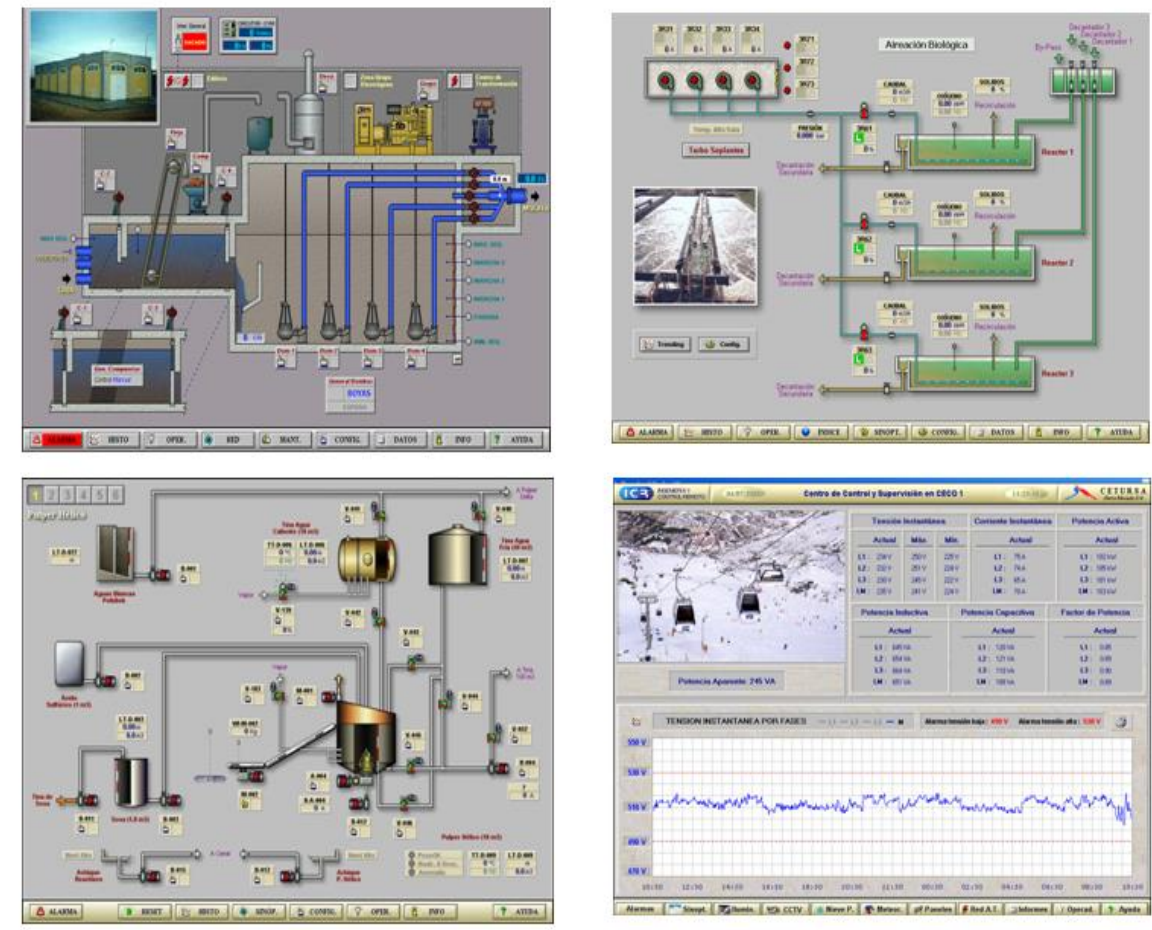

Figura 4. Ejemplos de supervisión de procesos mediante software SCADA

\subsection{Programa de prácticas}

Como siempre ocurre en asignaturas con una fuerte componente experimental, consideramos también que las prácticas de Informática Industrial son esenciales para la correcta formación del estudiante; incluso, se podría asegurar que gran parte de la materia teórica está dirigida a adquirir los conocimientos necesarios para realizar las 
prácticas, como objetivo didáctico último. El material que disponemos en el laboratorio requiere el desarrollo de varios seminarios específicos (no impartidos previamente en las clases teóricas) para que el alumno conozca en profundidad el equipamiento que va a utilizar.

Disponemos de los siguientes equipos físicos:

- Tarjetas Sensonic que incorpora dispositivos sensores y actuadores, sobre la cual el estudiante tendrá que realizar varios sistemas de control.

- Maqueta hidráulica.

- Maqueta de motores.

- Maqueta térmica.

- Conjunto de módulos conectados vía bus de campo, del fabricante ICP-DAS.

Por otro lado, disponemos de la herramienta de software SCADA Intouch del fabricante Wonderware, posiblemente el SCADA más utilizado en el mundo de control industrial. La tarjeta Sensonic se ha diseñado íntegramente entre el Departamento de Arquitectura y Tecnología de Computadores de la Universidad de Granada y la empresa Seven-Solutions, spin-off fundada también por profesores del mismo departamento.

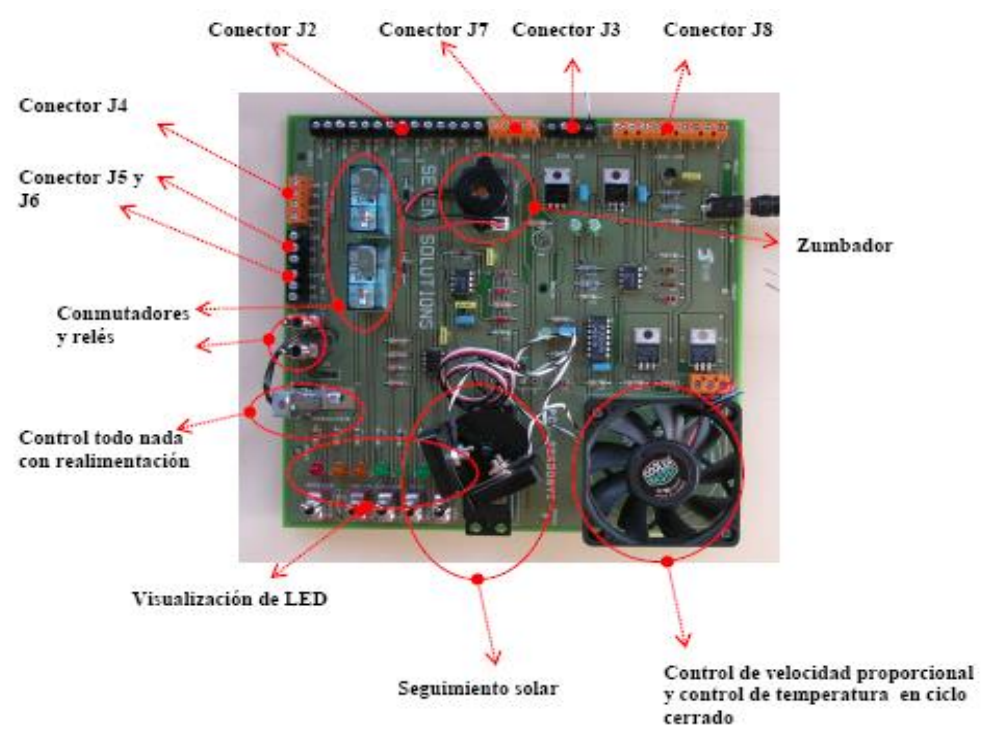

Figura 5. Fotografía de la tarjeta Sensonic

Con los elementos sensores incorporados, el estudiante puede realizar medidas continuas de temperatura, velocidad de giro de un motor, e intensidad luminosa en placas fotovoltáicas, además de detección de presencia mediante efecto Hall, y lectura de la posición de interruptores. Como elementos actuadores dispone de servomotor de corriente continua, solenoide lineal, zumbador, relés de potencia y calefactor de regulación continua. (Ver Figura 5). La tarjeta es de muy bajo coste y disponemos de 25 unidades para que el alumno pueda trabajar individualmente realizando 
controlador de tipo todo-nada, controladores PID discretos de temperatura, control digital de posición, y actuaciones de control secuencial.

Los sensores y actuadores de la tarjeta Sensonic se supervisan y controlan mediante un conjunto de módulos de la serie 7000 del fabricante ICP-DAS, conectados mediante un bus de campo propio sobre interfaz físico RS-485.

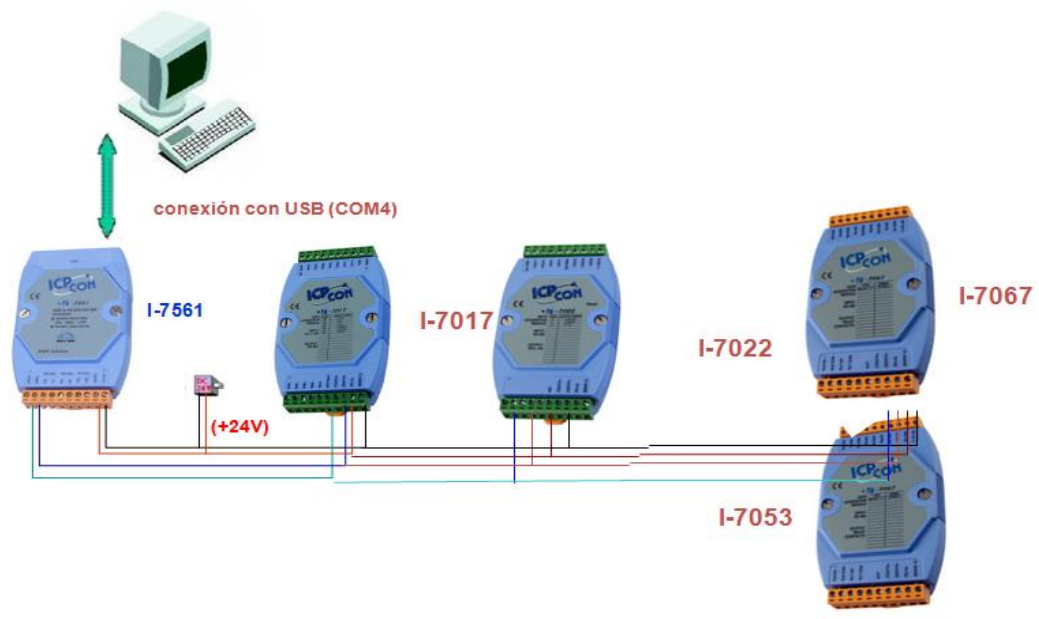

Figura 6. Módulos ICP-DAS

Los módulos de conversión A/D, D/A y de entradas y salidas digitales (ver Figura 6), se conectan vía USB con el ordenador que manejará el alumno para realizar el software de supervisión y control de la tarjeta Sensonic y de las restantes maquetas. Para ello utilizará el SCADA Intouch Wonderware, sin coste de licencias, siempre que no se utilicen más de 32 variables (tags) de entrada/salida.

Antes de empezar a controlar la tarjeta Sensonic, el alumno recibe un seminario didáctico de Intouch básico, para a continuación realizar una simulación libre de un proceso industrial, al objeto de familiarizarse con esta herramienta de configuración, que le permite realizar fácilmente esquemas sinópticos de procesos, gráficos de tendencias en tiempo real, históricos, alarmas, etc. (ver Figura 7).

A continuación se imparte un seminario sobre OPC, para posteriormente utilizar el servidor OPC de IPC-DAS y OPClink de Intouch. Una vez configurado el sistema, y creados los tags (variables de E/S), el objetivo siguiente es crear una aplicación SCADA para medir las magnitudes de todos los sensores y actuar sobre los elementos de control disponibles en la tarjeta Sensonic, tanto discretos como continuos.

Más adelante se plantean varios objetivos: a) control de temperatura todo-nada con histéresis, b) control PID de temperatura con algoritmo recursivo, c) ajuste experimental de constantes PID en función de la respuesta transitoria, d) control de servomecanismo y e) posicionamiento de mini-paneles fotovoltáicos para conseguir la máxima intensidad luminosa. 


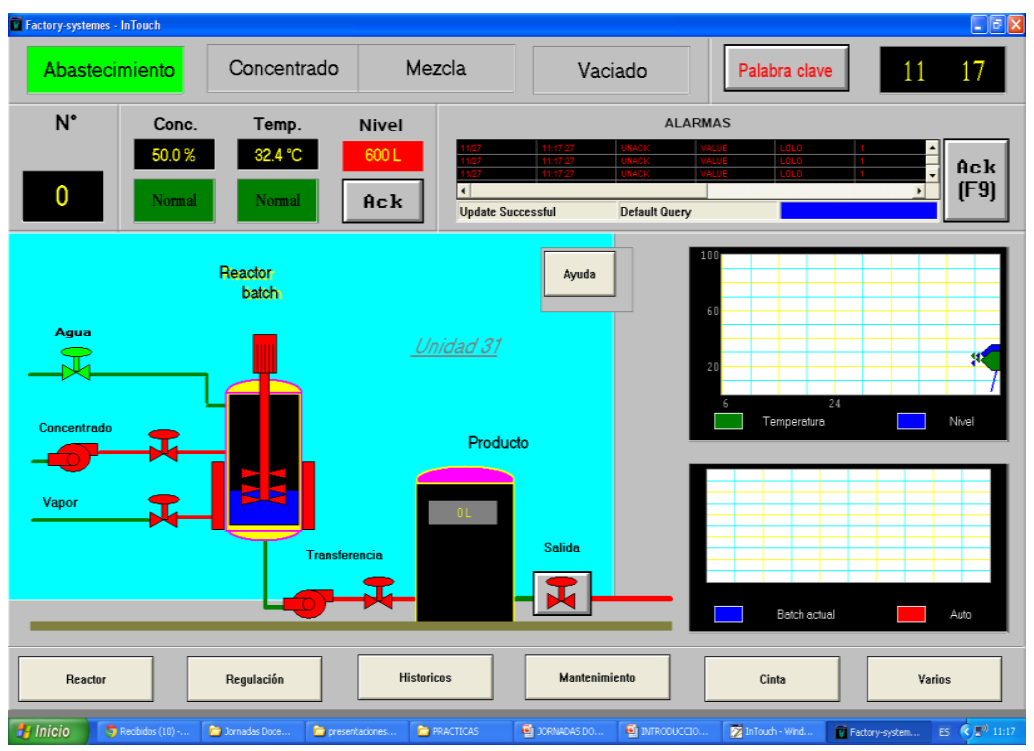

Figura 7. Pantalla ejemplo realizada con SCADA Intouch

Más adelante se plantean varios objetivos: a) control de temperatura todo-nada con histéresis, b) control PID de temperatura con algoritmo recursivo, c) ajuste experimental de constantes PID en función de la respuesta transitoria, d) control de servomecanismo y e) posicionamiento de mini-paneles fotovoltáicos para conseguir la máxima intensidad luminosa.

Disponemos también de tres maquetas (hidráulica, térmica y de motores) para prácticas opcionales y de demostración (ver Figura 8).

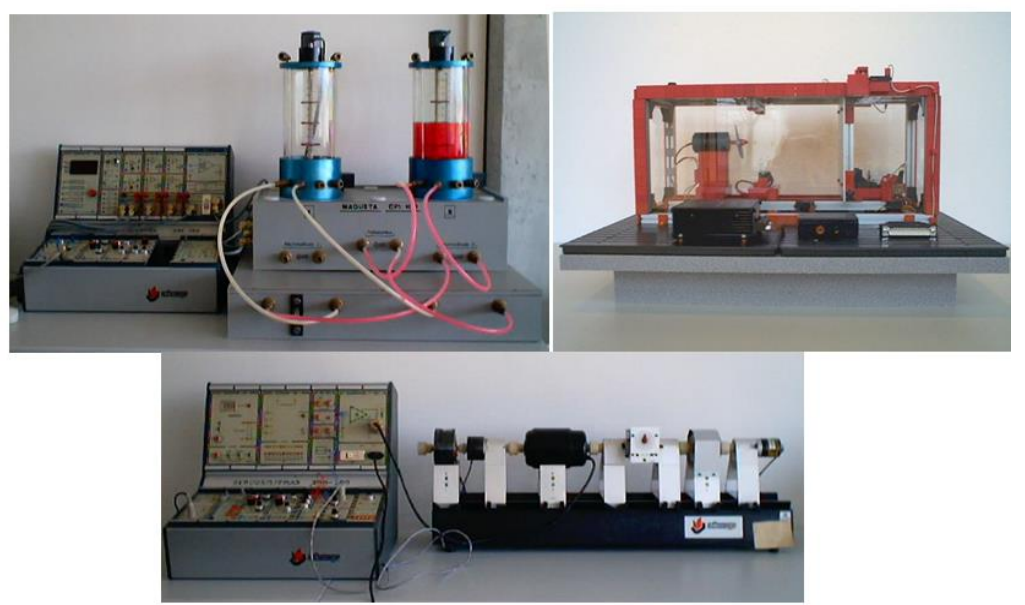

Figura 8. Maquetas hidráulica, térmica y de control de motores. 


\section{Conclusiones}

Se ha presentado en este artículo los objetivos y contenidos de la asignatura optativa "Informática Industrial" del Grado en Informática que se imparte en la Escuela Técnica Superior de Ingenierías Informática y de Telecomunicación de la Universidad de Granada. Esta asignatura, eminentemente práctica pretende transmitir al alumno los conocimientos suficientes para que el futuro profesional informático pueda integrarse en equipos multidisciplinares de empresas de Ingeniería de Control Industrial. Por experiencia en asignaturas precedentes, anteriores al plan de estudios actual, la mayoría de los alumnos tiene un gran interés, y tras las primeras clases se consigue un alto grado de motivación, que se incrementa cuando entra en el laboratorio y ve que con bastante facilidad realiza las prácticas satisfactoriamente. El estudiante agradece que, aunque los procesos a controlar se desarrollan sobre maquetas, las herramientas que utilizan son las que realmente se van a encontrar en el ámbito real, si finalmente desarrollan su vida profesional en el campo industrial.

Agradecimientos. Al Secretariado de Innovación Docente de la Universidad de Granada por los Proyectos de Innovación Docente concedidos que nos han permitido financiar la adquisición de algunas de las maquetas de laboratorio utilizadas en prácticas.

\section{Referencias}

1. M. Damas, J. Ortega, O. Baños: "Una iniciativa para la coordinación y difusión de la docencia del perfil de Ingeniería de Computadores del Grado de Informática de la UGR". Enseñanza y Aprendizaje de Ingeniería de Computadores. Revista de Experiencias Docentes en Ingeniería de Computadores. ISSN: 2173-8688, №2, pp.73-80, 2012.

2. S.A. Boyer, "SCADA: Supervisory Control and Data Acquisition", ISBN: 978-1556178771, ISA. 3 edition, 2004

3. W. Mahnke, S. Leitner, M. Damm, “OPC Unified Architecture”, ISBN: 978-3540688983, Springer, 1 edition, 2009.

4. M.Damas, H.Pomares, I.Rojas: "A new E-learning Strategy for Industrial Computation Students”. IASK International Conference Teaching and Learning, Aveiro (Portugal), 2008.

5. M.Damas, G.Olivares, H.Pomares, A.Olivares: "Laboratorio Virtual basado en Web para el ciclo completo de desarrollo de sistemas de control industriales". Workshop en Informática Industrial (WIIND), ISBN: 84-692-2381-9, Universidad Rey Juan Carlos, Madrid, 2009.

6. M.Damas, H.Pomares, G.Olivares: "Innovación en la docencia práctica de asignaturas relacionadas con la informática industrial". 1 $1^{a}$ Jornadas Andaluzas de Innovación Docente Universitaria. ISBN: 978-84-692-7263-3, Córdoba, 2009. 